\title{
Premature Aging of the Microcirculation in Patients with Advanced Chronic Kidney Disease
}

\author{
Oanh H.D. Thang ${ }^{a} \quad$ Erik H. Sernéb Muriel P.C. Grooteman ${ }^{a}$ \\ Yvo M. Smulders ${ }^{b}$ Piet M. ter Wee ${ }^{a}$ Geert-Jan Tangelderc Menso J. Nubéa \\ Departments of a Nephrology, ${ }^{\mathrm{b}}$ Internal Medicine, and ${ }^{\mathrm{C} P h y s i o l o g y, ~ I n s t i t u t e ~ f o r ~ C a r d i o v a s c u l a r ~ R e s e a r c h ~}$ \\ VU Medical Center, Amsterdam, The Netherlands
}

\section{Key Words}

Microcirculation · Iontophoresis · Endothelial function · Aging · Chronic kidney disease

\begin{abstract}
Background: Increasing age and advanced chronic kidney disease (CKD) are both associated with an attenuated vasodilator response of the skin microcirculation. In the present study, we investigated the effect of aging on microvascular reactivity in patients with advanced CKD. Methods: Acetylcholine (ACh)-mediated endothelium-dependent vasodilation and sodium nitroprusside (SNP)-mediated endothelium-independent vasodilation were assessed by iontophoresis combined with laser Doppler flowmetry. Microvascular function was compared between 52 patients with advanced CKD (stage 4-5: $n=16$; end-stage renal disease: $n=36$ ) and 33 healthy control subjects. As aging has an important effect on microvascular function, both control subjects and CKD patients were divided in subgroups younger and older than 45 years. Linear regression analysis was applied to assess potential associations between microvascular function and various demographic and clinical parameters. Results: There were three main findings. (1) In young patients with advanced CKD, both ACh- and SNP-mediated vasodilations were impaired if compared to young healthy controls ( $p=0.04$ and $p=0.056$, respectively). (2) In young patients with advanced CKD, microvascular function was similar to old healthy controls and elderly patients with advanced CKD. (3) Whereas age was inversely associated with microvascular function in healthy controls ( $\log$ ACh-mediated vasodilation $R=-0.41 ; p=0.02$ and log SNPmediated vasodilation $R=-0.38 ; p=0.03$ ), no such relation was found in patients with advanced CKD. Conclusions: Our results are consistent with premature aging of the microvascular vasodilatory capacity in patients with advanced CKD.

Copyright $\odot 2012$ S. Karger AG, Basel
\end{abstract}




\section{Introduction}

Cardiovascular morbidity and mortality are extremely high in patients with chronic kidney disease (CKD) [1]. Whereas classical risk factors, such as high blood pressure and cholesterol, contribute to cardiovascular disease in the early stages of CKD, non-traditional risk factors, including disorders of mineral metabolism, play a prevailing role in the vascular abnormalities of patients with advanced CKD [2].

In CKD not only the aorta and large conduit arteries may be affected but also the microcirculation, consisting of small arteries and arterioles, capillaries and venules [3]. In longstanding CKD, blood vessels are characterized by extensive calcifications in both the intimal and medial layers, leading to an increased vascular stiffening and loss of compliance. As a result, both the pulsatile component of blood pressure, as indicated by an augmented pulse pressure, and peripheral resistance, expressed as mean arterial pressure, are increased [4]. In the microcirculation, these changes may lead to an attenuated vasodilatory capacity and loss of capillary architecture and rarefaction [5]. Indeed, in patients with advanced CKD, it has recently been shown that the functional and structural number of capillaries, as analyzed by capillary nailfold microscopy, is decreased [6]. Blunted post-ischemic increase in skin blood flowmotion by laser Doppler tracing can be considered an early sign of microvascular endothelial dysfunction in CKD [7].

In addition to CKD, aging is also associated with an attenuated vasodilator response of the microcirculation to a variety of stimuli [8-12] which is largely the result of endothelial dysfunction. Accelerated vascular aging, associated with Klotho deficiency, is a characteristic of subjects with CKD [13-15]. Even in childhood, end-stage renal disease (ESRD) is associated with structural abnormalities in arterial wall properties, comparable with adult levels [16]. Research on vascular calcification and stiffness in CKD patients was focused mainly on coronary and other large arteries, but little is known about microcirculatory dysfunction.

In this study, the effect of age on endothelium-dependent and endothelium-independent vasoreactivity in the microcirculation, as assessed non-invasively by iontophoresis of acetylcholine (ACh) and sodium nitroprusside (SNP), respectively, was explored both in a group of healthy subjects and in a group of patients with advanced CKD.

\section{Patients and Methods}

\section{Patients and Controls}

A total of 52 patients [ 16 patients with CKD stage $4-5$ not yet on dialysis with an estimated glomerular filtration rate (eGFR) $<20 \mathrm{ml} / \mathrm{min}$ ], 20 hemodialysis (HD) and 16 peritoneal dialysis patients were recruited from the outpatient clinic and the dialysis unit of our hospital after giving informed consent. Thirty-three apparently healthy subjects served as controls. All study subjects were between 20 and 75 years old. Exclusion criteria were: life expectancy $<3$ months due to non-renal disease, diabetes mellitus, as defined by medical treatment for diabetes or a fasting glucose $>6.0 \mathrm{mmol} / \mathrm{l}$, and active auto-immune disease. The study protocol was approved by the local ethics committee.

\section{Laboratory Values}

Kidney function, expressed as eGFR normalized for $1.73 \mathrm{~m}^{2} \mathrm{BSA}$, was calculated by the Cockcroft and Gault formula in controls and by the mean of urine creatinine and urea clearances in CKD patients. Parameters of mineral metabolism (calcium corrected for albumin, phosphorus, intact parathyroid hormone) and routine laboratory parameters (hemoglobin, albumin and bicarbonate) were measured in patients with CKD. All blood samples were 
drawn in the fasting state. Intact parathyroid hormone levels were measured by a commercially available immunometric assay (Luminescence Architect; Abbott Laboratories, Chicago, Ill., USA).

\section{Clinical Characteristics}

Clinical history and medication use were assessed by information from medical charts. Body mass index (BMI) was calculated from weight and height measurements. Patients were considered to have a history of cardiovascular events if there was a previous stroke or cardiac event, including myocardial infarction, congestive heart failure and angina, or clinical peripheral vascular disease. The causes of renal disease are described in table 2.

\section{Iontophoresis}

Endothelium-(in)dependent vasodilation of finger skin microcirculation was evaluated by iontophoresis in combination with laser Doppler flowmetry [17]. Laser Doppler flowmetry is a non-invasive method to measure skin perfusion. A laser beam penetrates the skin and a fraction of the light is backscattered by moving blood objects and undergoes a frequency shift according to the Doppler principle, generating a signal proportional to tissue perfusion. Laser Doppler flux was measured on the middle phalanx of the third and fourth finger on the nondominant hand with the Periflux 4000 system (Perimed) and expressed as arbitrary perfusion units. In HD patients, measurements were obtained from the contralateral shunt arm. HD patients were treated twice or thrice weekly and analyzed on a midweek non-dialysis day. Iontophoresis is a non-invasive method of drug application that allows the local transfer of charged substances across the skin by use of a small electric current. A battery-powered iontophoresis controller (Phoresor II; Iomed) was used to provide a direct current for drug iontophoresis. ACh (1\%, Miochol; IOLAB, Bournonville Pharma) was delivered with an anodal current on the middle finger: 7 doses $(0.1 \mathrm{~mA}$ for $20 \mathrm{~s})$ were delivered, with a 60 -second interval between each dose. A 60-second interval between each iontophoresis period was required to achieve the plateau of the response following each delivery of ACh. Day-to-day variation coefficient of the relative increase from baseline to the final two deliveries was $9.8 \pm 5.6 \%$ as determined in 9 subjects on two occasions. SNP $(0.01 \%$, Nipride; Roche) was delivered with a cathodal current; 9 doses $(0.2 \mathrm{~mA}$ for $20 \mathrm{~s})$ were delivered, with a 120 -second interval between each dose. A 120-second interval between each iontophoresis period was required to achieve the plateau of the response after each delivery of SNP. Day-to-day variation coefficient of the relative increase from baseline to the final two deliveries was $8.3 \pm 5.4 \%$ as determined in 9 subjects on two occasions. Because the responses to vehicle are known to be negligible compared with responses to ACh and SNP, we refrained from doing vehicle control studies.

ACh-mediated and SNP-mediated vasodilations were defined as percentage increase from baseline to the final $2 \mathrm{~min}$ of the plateau phase. Additional analyses were performed by measuring the absolute increase in perfusion units and by measuring the area under the response curve for ACh and SNP.

\section{Statistical Analysis}

Variables are presented as mean \pm SD or as median, 10th-90th percentile and interquartile range. When appropriate, data with a skewed distribution were log-transformed. ANOVA was used to compare differences between healthy controls and CKD patients. Nonparametric tests were used in the case of small numbers or abnormally distributed data sets. Linear regression analysis was applied to explore various demographic and clinical determinants of ACh- or SNP-mediated vasodilation. A p value of $<0.05$ was considered statistically significant. All analyses were performed with the statistical software package SPSS version 18.0 (SPSS Inc., Chicago, Ill., USA). 
Table 1. Demographic variables

\begin{tabular}{|c|c|c|c|}
\hline & Healthy controls & CKD stage $4-5$ & ESRD \\
\hline Number (male/female) & $33(19 / 14)$ & $16(10 / 6)$ & $36(28 / 8)$ \\
\hline Mean age $\pm S D$, years & $49.5 \pm 16.7$ & $56.9 \pm 11.5$ & $55.0 \pm 13.9$ \\
\hline BMI & $24.3 \pm 2.7$ & $25.9 \pm 4.3$ & $23.9 \pm 3.4$ \\
\hline Systolic blood pressure, mm Hg & $126 \pm 17^{*}$ & $148 \pm 30^{*}$ & $139 \pm 25$ \\
\hline Diastolic blood pressure, $\mathrm{mm} \mathrm{Hg}$ & $79 \pm 9$ & $85 \pm 16$ & $80 \pm 10$ \\
\hline $\mathrm{eGFR}, \mathrm{ml} / \mathrm{min} / 1.73 \mathrm{~m}^{2}$ & $90[76-108]$ & $11[8-13]$ & $4[0-6]$ \\
\hline History of cardiovascular disease, n (\%) & 0 & $4(25)$ & $9(25)$ \\
\hline \multicolumn{4}{|l|}{ Dialysis vintage, years } \\
\hline Peritoneal dialysis patients $(n=16)$ & & & $1.76 \pm 1.06$ \\
\hline Hemodialysis patients $(\mathrm{n}=20)$ & & & $2.20 \pm 2.51$ \\
\hline Smoking, n (\%) & 0 & $8(50)$ & $18(50)$ \\
\hline \multicolumn{4}{|l|}{ Medication } \\
\hline Diuretic use, n (\%) & & $7(44)$ & $18(50)$ \\
\hline Inhibitors of RAAS, $\mathrm{n}(\%)$ & & $13(81)$ & $25(69)$ \\
\hline Calcium antagonist, $\mathrm{n}(\%)$ & & $10(63)$ & $12(33)$ \\
\hline Beta-blocker, n (\%) & & $8(50)$ & $23(64)$ \\
\hline Calcium carbonate, n (\%) & & $4(25)$ & $22(61)$ \\
\hline Sevelamer, n (\%) & & $5(31)$ & $25(69)$ \\
\hline Lanthanum carbonate, $\mathrm{n}(\%)$ & & 0 & $9(25)$ \\
\hline
\end{tabular}

Data are presented as mean \pm SD or as median [interquartile range]. RAAS $=$ Renin angiotensin aldosterone system. ${ }^{*} \mathrm{p}<0.01$ healthy controls vs. CKD stage $4-5$.

\section{Results}

Demographic, Clinical and Laboratory Characteristics

Baseline characteristics of patients and controls are shown in table 1. Control subjects were all non-smokers and did not use any medication. Median eGFR in healthy controls was $90 \mathrm{ml} / \mathrm{min} / 1.73 \mathrm{~m}^{2}$, in CKD stage $4-5$ patients $11 \mathrm{ml} / \mathrm{min} / 1.73 \mathrm{~m}^{2}$ and in ESRD patients $4 \mathrm{ml} / \mathrm{min} / 1.73 \mathrm{~m}^{2}$. Dialysis vintage of peritoneal dialysis patients was not significantly different from HD patients (1.76 \pm 1.06 and $2.20 \pm 2.51$ years, respectively). ACh-mediated and SNP-mediated vasodilations were similar in these groups. Systolic blood pressure was significantly higher in CKD stage 4-5 patients than in healthy controls. The mean ages in healthy controls, CKD stage 4-5 and ESRD patients were $49.5 \pm 16.7,56.9 \pm 11.5$ and 55.0 \pm 13.9 years, respectively (table 1 ). As age is an important determinant of microvascular function [18], and it was different between control subjects and advanced CKD patients, a subdivision was made according to age. The primary goal was to create subgroups with identical 'old age' amongst healthy controls and patients with advanced CKD. For this purpose, the division between 'old' and 'young' was arbitrarily set at 45 years. The mean ages in the group of old healthy controls and old CKD patients were $60.9 \pm 7.1$ and $61.1 \pm 7.8$ years, respectively (table 2). In both 'young' groups, age was a bit less well matched (controls 29.5 \pm 5.4 years, CKD patients $34.9 \pm 7.2$ years). Laboratory values of the patients, including markers of mineral metabolism, are shown in table 2 .

\section{Microvascular Function}

Comparison between Healthy Controls and Patients with Advanced CKD

Both ACh- and SNP-mediated vasodilations were similar in CKD stage 4-5 and ESRD patients [median (interquartile range) Ach-mediated vasodilations $287(113-1,226)$ and 313 
Thang et al.: Premature Aging of the Microcirculation in Patients with Advanced Chronic

Kidney Disease

Table 2. Demographic variables: groups were split into subgroups consisting of persons over and under 45 years

\begin{tabular}{|c|c|c|c|c|}
\hline & $\begin{array}{l}\text { Young healthy } \\
\text { controls } \\
\text { (<45 years) }\end{array}$ & $\begin{array}{l}\text { Young } \\
\text { advanced CKD } \\
(<45 \text { years })\end{array}$ & $\begin{array}{l}\text { Old healthy } \\
\text { controls } \\
\text { ( } \geq 45 \text { years) }\end{array}$ & $\begin{array}{l}\text { Old advanced } \\
\text { CKD } \\
\text { ( } \geq 45 \text { years })\end{array}$ \\
\hline Number (male/female) & $12(7 / 5)$ & $11(8 / 3)$ & $21(12 / 9)$ & $41(30 / 11)$ \\
\hline Mean age $\pm S D$, years & $29.5 \pm 5.4$ & $34.9 \pm 7.2$ & $60.9 \pm 7.1$ & $61.1 \pm 7.8$ \\
\hline BMI & $23.7 \pm 2.3$ & $22.8 \pm 4.7$ & $24.7 \pm 3.0$ & $25.0 \pm 3.4$ \\
\hline Systolic blood pressure, mm Hg & $113 \pm 13^{\#}$ & $128 \pm 20$ & $134 \pm 15^{\#}$ & $145 \pm 27^{\#}$ \\
\hline Diastolic blood pressure, $\mathrm{mm} \mathrm{Hg}$ & $73 \pm 8^{\#}$ & $77 \pm 10$ & $83 \pm 7^{\#}$ & $83 \pm 13^{\#}$ \\
\hline $\mathrm{eGFR}, \mathrm{ml} / \mathrm{min}$ & $116[96-121]$ & $5[0-7]$ & $85[7-98]$ & $6[3-12]$ \\
\hline \multicolumn{5}{|l|}{ Cause of renal disease } \\
\hline Hypertension, n (\%) & & $5(46)$ & & $23(56)$ \\
\hline Hereditary disease, $\mathrm{n}(\%)$ & & $3(27)$ & & $10(24)$ \\
\hline Immune-complex glomerulonephritis, $\mathrm{n}(\%)$ & & 0 & & $5(12)$ \\
\hline Others, $\mathrm{n}(\%)$ & & $3(27)$ & & $3(7)$ \\
\hline History of cardiovascular disease, n (\%) & 0 & 0 & 0 & $13(32)$ \\
\hline Smoking, n (\%) & 0 & $4(36)$ & 0 & $22(54)$ \\
\hline \multicolumn{5}{|l|}{ Laboratory values } \\
\hline Calcium, mmol/l & & $2.31 \pm 0.21$ & & $2.39 \pm 0.14$ \\
\hline Phosphorus, mmol/l & & $1.74 \pm 0.44$ & & $1.62 \pm 0.39$ \\
\hline Albumin, $g / 1$ & & $38 \pm 3.5$ & & $37.5 \pm 3.7$ \\
\hline Parathyroid hormone, $\mathrm{pmol} / \mathrm{l}$ & & $17(1-69)$ & & $26(17-37)$ \\
\hline Bicarbonate, $\mathrm{mmol} / \mathrm{l}$ & & $24.1 \pm 4.7$ & & $22.6 \pm 3.0$ \\
\hline Hemoglobin, mmol/l & & $7.5 \pm 0.9$ & & $7.4 \pm 0.9$ \\
\hline
\end{tabular}

Data are presented as mean $\pm \mathrm{SD}$ or as median [interquartile range]. Reference values are in braces. ${ }^{\#} \mathrm{p}<0.05$ young healthy controls vs. old healthy controls/old advanced CKD patients.

Table 3. Correlations between demographic and clinical parameters with microvascular function (log ACh-mediated and log SNP-mediated vasodilation) by linear regression analysis in healthy controls $(\mathrm{n}=33)$

\begin{tabular}{lcccr}
\hline Determinant & $\mathrm{B}$ & $95 \%$ CI & $\mathrm{p}$ & $\mathrm{r}$ \\
\hline log ACh-mediated vasodilation & & & & \\
Age, years & -0.01 & -0.02 to -0.002 & $\mathbf{0 . 0 2}$ & -0.41 \\
Female gender & -0.09 & -0.41 to 0.24 & 0.58 & -0.10 \\
eGFR, ml/min & 0.01 & 0.002 to 0.02 & $\mathbf{0 . 0 2}$ & 0.44 \\
BMI & -0.03 & -0.09 to 0.03 & 0.31 & -0.18 \\
Systolic blood pressure, mm Hg & -0.01 & -0.02 to -0.001 & $\mathbf{0 . 0 2}$ & -0.39 \\
Diastolic blood pressure, mm Hg & -0.01 & -0.03 to 0.01 & 0.30 & -0.19 \\
\hline log SNP-mediated vasodilation & & & & \\
Age, years & -0.01 & -0.02 to -0.001 & $\mathbf{0 . 0 3}$ & -0.38 \\
Female gender & 0.31 & 0.05 to 0.57 & $\mathbf{0 . 0 2}$ & 0.40 \\
eGFR, ml/min & 0.01 & -0.002 to 0.01 & 0.13 & 0.29 \\
BMI & -0.02 & -0.07 to 0.03 & 0.38 & -0.16 \\
Systolic blood pressure, mm Hg & -0.01 & -0.02 to -0.004 & $\mathbf{0 . 0 0 3}$ & -0.50 \\
Diastolic blood pressure, mm Hg & -0.02 & -0.03 to -0.002 & 0.03 & -0.39 \\
\hline
\end{tabular}


Fig. 1. ACh- and SNP-mediated vasodilations, defined as percentage increase from baseline to the final $2 \mathrm{~min}$ of the plateau phase. A box represents the 25 th75th percentile range, the whiskers represent the 10 th-90th percentile range and the line within the boxes the median value.
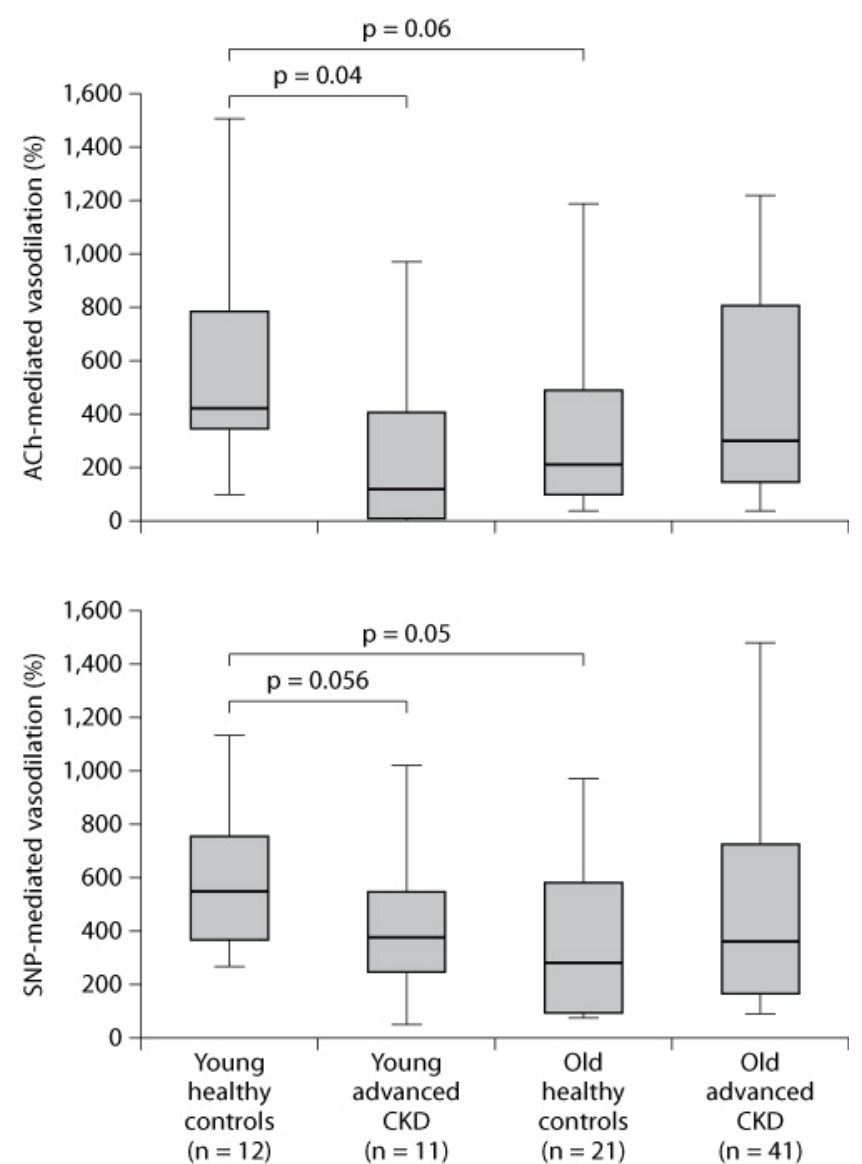

(158-781), respectively, $\mathrm{p}=0.79$; SNP-mediated vasodilations 396 (191-730) and 333 (125$744)$, respectively, $\mathrm{p}=0.75]$. Therefore, these groups were pooled and denoted 'advanced CKD'. From our analysis it appeared, first, that ACh-mediated and SNP-mediated vasodilations in young patients with advanced CKD were impaired, if compared with young healthy controls. Second, microvascular function in young patients with advanced CKD is similar to both old healthy controls and elderly patients with advanced CKD (fig. 1). Similar results were obtained by measuring the percentage increase from baseline to the final $2 \mathrm{~min}$ of the plateau phase, the absolute increase in perfusion units, or by measuring the area under the response curve for both ACh and SNP (data not shown).

Correlations between Microvascular Function, and Demographic and Clinical

Variables in Healthy Controls

In healthy controls, linear regression analysis demonstrated that age and systolic blood pressure were inversely correlated with log ACh-mediated vasodilation, while eGFR showed a positive correlation (table 3 ). With respect to log SNP-mediated vasodilation, age and systolic blood pressure were inversely and female gender positively associated. 
Thang et al.: Premature Aging of the Microcirculation in Patients with Advanced Chronic

Kidney Disease

Table 4. Associations between demographic and clinical parameters with microvascular function (log ACh-mediated and log SNP-mediated vasodilation) by linear regression analysis in patients with advanced CKD $(\mathrm{n}=52)$

\begin{tabular}{lcccr}
\hline Determinant & $\mathrm{B}$ & $95 \% \mathrm{CI}$ & $\mathrm{p}$ & $\mathrm{r}$ \\
\hline log ACh-mediated vasodilation & & & & \\
Age, years & 0.02 & $0.00-0.03$ & $\mathbf{0 . 0 5}$ & 0.28 \\
Female gender & 0.15 & $-0.29-0.59$ & 0.49 & 0.10 \\
eGFR, ml/min & 0.01 & $-0.03-0.05$ & 0.57 & 0.08 \\
BMI & -0.04 & $-0.09-0.01$ & 0.09 & -0.24 \\
Systolic blood pressure, mm Hg & 0.01 & $-0.001-0.01$ & 0.12 & 0.22 \\
Diastolic blood pressure, mm Hg & 0.02 & $0.01-0.04$ & $\mathbf{0 . 0 0 3}$ & 0.41 \\
Phosphate, mmol/l & -0.26 & $-0.75-0.23$ & 0.30 & -0.15 \\
Calcium, mmol/l & 0.65 & $-0.56-1.87$ & 0.28 & 0.15 \\
\hline log SNP-mediated vasodilation & & & & -0.05 \\
Age, years & -0.002 & $-0.01-0.01$ & 0.74 & 0.04 \\
Female gender & 0.04 & $-0.25-0.33$ & 0.80 & 0.09 \\
eGFR, ml/min & 0.01 & $-0.02-0.04$ & 0.53 & -0.23 \\
BMI & -0.03 & $-0.06-0.01$ & 0.10 & -0.03 \\
Systolic blood pressure, mm Hg & 0.00 & $-0.01-0.004$ & 0.84 & 0.07 \\
Diastolic blood pressure, mm Hg & 0.003 & $-0.01-0.01$ & 0.61 & -0.01 \\
Phosphate, mmol/l & -0.01 & $-0.34-0.31$ & 0.95 & -0.17 \\
Calcium, mmol/l & -0.48 & $-1.27-0.31$ & 0.23 & \\
\hline
\end{tabular}

Correlations between Microvascular Function, and Demographic and Clinical Variables in Patients with CKD

In patients with advanced $\mathrm{CKD}$, linear regression analysis showed that age and diastolic blood pressure were positively correlated with log ACh-mediated vasodilation (table 4). There were no correlations between calcium and phosphate levels, and Ach-mediated or SNP-mediated vasodilation.

\section{Discussion}

Increasing age and advanced CKD are both associated with an attenuated vasodilator response of the skin microcirculation [8-12]. However, whether aging impairs endotheliumdependent vasoreactivity in CKD patients even further is presently unknown. Our study demonstrates that microvascular function, as assessed with laser Doppler in combination with the iontophoretic delivery of ACh and SNP onto the skin, was inversely associated with age in healthy individuals, but not in patients with advanced CKD. Young patients with advanced CKD showed impaired vasodilation compared to young healthy controls. No differences could be observed between young patients with advanced CKD and both old healthy controls and elderly patients with CKD. Together, these data are compatible with the concept of premature aging of the vasculature in patients with CKD $[16,19]$.

In previous reports, microvascular dysfunction has been extensively described, not only in patients with diabetes mellitus [20,21], hypertension [22] and coronary heart disease [23], but also in patients with CKD $[24,25]$. In patients with advanced CKD, we recently demonstrated substantial capillary rarefaction both at baseline, after hyperemic stimulation and after venous occlusion [6]. Together these alterations suggested not only a functional, but also a structural disorder of the microcirculation in the skin of these patients. Klotho deficiency 
is associated with hyperphosphatemia-induced accelerated aging. Decreased Klotho expression is supposed to be the initiator of CKD-MBD pathophysiology [13-15]. As cutaneous capillary rarefaction was strongly and inversely correlated with serum phosphate levels [6], and high phosphate levels in CKD patients are associated with calcifications in the media of large and medium-size blood vessels [26, 27], calcified depositions in the subendothelial layers of the microcirculation may contribute to these findings [6,28]. In the present analysis, we speculated that these pathophysiological changes would lead to deficient endothelium-dependent and endothelium-independent responses to locally administered vasodilatory stimuli.

In patients with CKD, both a blunted and a normal response to ACh and SNP have been described. However, in publications showing a reduced response, the control group was either younger, hypertensive or incompletely described $[24,29,30]$. When normotensive CKD patients were compared with age-matched controls [31], no marked differences were found. Actually, aging per se appeared to be associated with reduced skin reactivity, as elderly persons showed a lower dose-response curve after both ACh [32] and insulin [33]. By contrast, in comparison with age-matched controls, both thermal [25,34] and reactive hyperemia [25] in the volar forearm were considerably reduced in patients with CKD, and even more in the presence of cardiovascular disease and diabetes mellitus [25]. Whether differences in methodology - thermal and reactive hyperemia versus the iontophoretic delivery of ACh and SNP - or dissimilar demographic and/or clinical characteristics explain the contrasting findings in patients with advanced CKD is not readily apparent from these studies.

Our study confirms and extends previous findings that age influences the vasoreactivity of the microcirculation in both the kidney and the skin of healthy subjects [30,35]. With respect to microvascular function, our study suggests that premature aging occurs in young patients with advanced CKD. As differences were not found between old healthy controls and elderly patients with advanced CKD, it appears that age itself is of paramount importance for the observed decrease in microvascular reactivity. The presence of uremia, as manifested by various laboratory abnormalities, including disorders of mineral metabolism, did not aggravate these alterations. Hence, it appears that in the elderly, the uremic state itself has no additional negative influence on microvascular reactivity in skin on top of the alterations induced by aging itself.

Remarkably, in patients with advanced CKD, linear regression analysis demonstrated that older age and higher diastolic blood pressure were slightly associated with preserved AChmediated vasodilation. In this respect, it is interesting to note that elderly adults with low diastolic blood pressure have a higher risk of death [36]. Apart from survival bias, the long-lasting use of vasoactive medication, such as ACE inhibitors or beta-blockers, may contribute to these findings. The design of our study, however, does not permit such a far-reaching conclusion.

Interestingly, in healthy insulin-sensitive overweight individuals, it was recently found that both endothelial-dependent and independent cutaneous vasodilations were undisturbed, despite marked capillary rarefaction in these patients [37]. Hence, capillary rarefaction may occur independently from microcirculatory dysfunction, not only in obese diabetic patients but also in patients with advanced CKD.

Our study has important limitations. First, as many patients with advanced CKD are treated with anti-hypertensive and phosphorus-binding medication, it is conceivable that these drugs influenced endothelium-dependent and endothelium-independent vasodilations. Second, whereas tobacco use was an exclusion criterion in the control group, patients who smoked were not excluded. Defining smoking as an exclusion criterion, in addition to diabetes and auto-immune diseases, would have precluded a substantial amount of patients from the study. Third, as the group of patients with advanced CKD consisted of individuals with CKD stage 4-5 and ESRD, our data must be interpreted with caution due to the heterogeneity of this group. 
To summarize, whereas age was inversely associated with microvascular function in healthy individuals, no such correlation was found in patients with advanced CKD. In young patients with advanced CKD, endothelium-dependent and endothelium-independent vasodilations, as measured by the iontophoretic delivery of ACh and SNP onto the skin, were impaired if compared to young healthy controls. As microvascular reactivity in young patients with advanced CKD was similar to old healthy controls and old patients with CKD, premature aging may underlie these findings.

\section{Acknowledgment}

This study was supported by Baxter Healthcare Corporation.

\section{References}

1 Foley RN, Parfrey PS, Sarnak MJ: Clinical epidemiology of cardiovascular disease in chronic renal disease. Am J Kidney Dis 1998;32:S112-S119.

-2 Block G, Port FK: Calcium phosphate metabolism and cardiovascular disease in patients with chronic kidney disease. Semin Dial 2003;16:140-147.

-3 Moe SM, Chen NX: Calciphylaxis and vascular calcification: a continuum of extra-skeletal osteogenesis. Pediatr Nephrol 2003;18:969-975.

-4 Mitchell GF, Vita JA, Larson MG, Parise H, Keyes MJ, Warner E, Vasan RS, Levy D, Benjamin EJ: Cross-sectional relations of peripheral microvascular function, cardiovascular disease risk factors, and aortic stiffness: the Framingham Heart Study. Circulation 2005;112:3722-3728.

-5 Fliser D, Wiecek A, Suleymanlar G, Ortiz A, Massy ZA, Lindholm B, Martinez-Castelao A, Agarwal R, Jager K, Dekker FW, Blankestijn PJ, Goldsmith DJ, Covic A, London G, Zoccali C: The dysfunctional endothelium in CKD and in cardiovascular disease: mapping the origin(s) of cardiovascular problems in CKD and of kidney disease in cardiovascular conditions for a research agenda. Kidney Int Sup 2011;1:6-9.

-6 Thang OH, Serne EH, Grooteman MP, Smulders YM, ter Wee PM, Tangelder GJ, Nube MJ: Capillary rarefaction in advanced chronic kidney disease is associated with high phosphorus and bicarbonate levels. Nephrol Dial Transplant 2011;26:3529-3536.

7 Rossi M, Cupisti A, Di Maria C, Galetta F, Barsotti G, Santoro G: Blunted post-ischemic increase of the endothelial skin blood flowmotion component as early sign of endothelial dysfunction in chronic kidney disease patients. Microvasc Res 2008;75:315-322.

8 Hagisawa S, Barbenel JC, Kenedi RM: Influence of age on postischaemic reactive hyperaemia. Clin Phys Physiol Meas 1991;12:227-237.

-9 Tew GA, Klonizakis M, Saxton JM: Effects of ageing and fitness on skin-microvessel vasodilator function in humans. Eur J Appl Physiol 2010;109:173-181.

-10 Minson CT, Holowatz LA, Wong BJ, Kenney WL, Wilkins BW: Decreased nitric oxide- and axon reflex-mediated cutaneous vasodilation with age during local heating. J Appl Physiol 2002;93:16441649.

-11 Holowatz LA, Houghton BL, Wong BJ, Wilkins BW, Harding AW, Kenney WL, Minson CT: Nitric oxide and attenuated reflex cutaneous vasodilation in aged skin. Am J Physiol Heart Circ Physiol 2003;284:H1662-H1667.

12 James MA, Tullett J, Hemsley AG, Shore AC: Effects of aging and hypertension on the microcirculation. Hypertension 2006;47:968-974.

13 Kuro O: Phosphate and Klotho. Kidney Int Suppl 2011;121:S20-S23.

-14 Hu MC, Shi M, Zhang J, Quinones H, Griffith C, Kuro-o M, Moe OW: Klotho deficiency causes vascular calcification in chronic kidney disease. J Am Soc Nephrol 2011;22:124-136.

-15 John GB, Cheng CY, Kuro-o M: Role of Klotho in aging, phosphate metabolism, and CKD. Am J Kidney Dis 2011;58:127-134. 
Thang et al.: Premature Aging of the Microcirculation in Patients with Advanced Chronic Kidney Disease

-16 Covic A, Mardare N, Gusbeth-Tatomir P, Brumaru O, Gavrilovici C, Munteanu M, Prisada O, Goldsmith DJ: Increased arterial stiffness in children on haemodialysis. Nephrol Dial Transplant 2006; 21:729-735.

-17 Serne EH, Stehouwer CD, ter Maaten JC, ter Wee PM, Rauwerda JA, Donker AJ, Gans RO: Microvascular function relates to insulin sensitivity and blood pressure in normal subjects. Circulation 1999; 99:896-902.

- 18 Holowatz LA, Thompson-Torgerson CS, Kenney WL: The human cutaneous circulation as a model of generalized microvascular function. J Appl Physiol 2008;105:370-372.

-19 Stenvinkel P: Chronic kidney disease: a public health priority and harbinger of premature cardiovascular disease. J Intern Med 2010;268:456-467.

-20 Morris SJ, Shore AC, Tooke JE: Responses of the skin microcirculation to acetylcholine and sodium nitroprusside in patients with NIDDM. Diabetologia 1995;38:1337-1344.

-21 Beer S, Feihl F, Ruiz J, Juhan-Vague I, Aillaud MF, Wetzel SG, Liaudet L, Gaillard RC, Waeber B: Comparison of skin microvascular reactivity with hemostatic markers of endothelial dysfunction and damage in type 2 diabetes. Vasc Health Risk Manag 2008;4:1449-1458.

-22 Lindstedt IH, Edvinsson ML, Edvinsson L: Reduced responsiveness of cutaneous microcirculation in essential hypertension - a pilot study. Blood Press 2006;15:275-280.

-23 Ijzerman RG, de Jongh RT, Beijk MA, van Weissenbruch MM, Delemarre-van de Waal HA, Serne $\mathrm{EH}$, Stehouwer CD: Individuals at increased coronary heart disease risk are characterized by an impaired microvascular function in skin. Eur J Clin Invest 2003;33:536-542.

-24 Cupisti A, Rossi M, Placidi S, Caprioli R, Morelli E, Vagheggini G, Barsotti G: Responses of the skin microcirculation to acetylcholine and to sodium nitroprusside in chronic uremic patients. Int J Clin Lab Res 2000;30:157-162.

-25 Kruger A, Stewart J, Sahityani R, O’Riordan E, Thompson C, Adler S, Garrick R, Vallance P, Goligorsky MS: Laser Doppler flowmetry detection of endothelial dysfunction in end-stage renal disease patients: correlation with cardiovascular risk. Kidney Int 2006;70:157-164.

-26 Moe SM, Chen NX: Mechanisms of vascular calcification in chronic kidney disease. J Am Soc Nephrol 2008;19:213-216.

-27 Cannata-Andia JB, Rodriguez-Garcia M, Carrillo-Lopez N, Naves-Diaz M, Diaz-Lopez B: Vascular calcifications: pathogenesis, management, and impact on clinical outcomes. J Am Soc Nephrol 2006; 17:S267-S273.

-28 Ichimaru K, Horie A: Microangiopathic changes of subepidermal capillaries in end-stage renal failure. Nephron 1987;46:144-149.

-29 Sigrist MK, McIntyre CW: Vascular calcification is associated with impaired microcirculatory function in chronic haemodialysis patients. Nephron Clin Pract 2008;108:c121-c126.

- 30 Farkas K, Nemcsik J, Kolossvary E, Jarai Z, Nadory E, Farsang C, Kiss I: Impairment of skin microvascular reactivity in hypertension and uraemia. Nephrol Dial Transplant 2005;20:1821-1827.

- 31 Cupisti A, Rossi M, Placidi S, Fabbri A, Morelli E, Vagheggini G, Meola M, Barsotti G: Responses of the skin microcirculation to acetylcholine in patients with essential hypertension and in normotensive patients with chronic renal failure. Nephron 2000;85:114-119.

-32 Rossi M, Cupisti A, Mariani S, Santoro G, Pentimone F: Endothelium-dependent and endotheliumindependent skin vasoreactivity in the elderly. Aging Clin Exp Res 2002;14:343-346.

-33 Rossi M, Cupisti A, Ricco R, Santoro G, Pentimone F, Carpi A: Skin vasoreactivity to insulin iontophoresis is reduced in elderly subjects and is absent in treated non-insulin-dependent diabetes patients. Biomed Pharmacother 2004;58:560-565.

-34 Stewart J, Kohen A, Brouder D, Rahim F, Adler S, Garrick R, Goligorsky MS: Noninvasive interrogation of microvasculature for signs of endothelial dysfunction in patients with chronic renal failure. Am J Physiol Heart Circ Physiol 2004;287:H2687-H2696.

- 35 Hollenberg NK, Adams DF, Solomon HS, Rashid A, Abrams HL, Merrill JP: Senescence and the renal vasculature in normal man. Circ Res 1974;34:309-316.

-36 Guichard JL, Desai RV, Ahmed MI, Mujib M, Fonarow GC, Feller MA, Ekundayo OJ, Bittner V, Aban IB, White M, Aronow WS, Love TE, Bakris GL, Zieman SJ, Ahmed A: Isolated diastolic hypotension and incident heart failure in older adults. Hypertension 2011;58:895-901.

- 37 Czernichow S, Greenfield JR, Galan P, Bastard JP, Charnaux N, Samaras K, Safar ME, Blacher J, Hercberg S, Levy BI: Microvascular dysfunction in healthy insulin-sensitive overweight individuals. J Hypertens 2010;28:325-332. 